\title{
Global Quantum Thermometry
}

\author{
Jesús Rubio $\odot^{1, *}$ Janet Anders $\odot{ }^{1,2}$ and Luis A. Correa $\odot^{1}$ \\ ${ }^{1}$ Department of Physics and Astronomy, University of Exeter, Stocker Road, Exeter EX4 4QL, United Kingdom \\ ${ }^{2}$ Institut für Physik und Astronomie, University of Potsdam, 14476 Potsdam, Germany
}

(Received 10 December 2020; revised 21 June 2021; accepted 27 September 2021; published 2 November 2021)

\begin{abstract}
A paradigm shift in quantum thermometry is proposed. To date, thermometry has relied on local estimation, which is useful to reduce statistical fluctuations once the temperature is very well known. In order to estimate temperatures in cases where few measurement data or no substantial prior knowledge are available, we build instead a method for global quantum thermometry. Based on scaling arguments, a mean logarithmic error is shown here to be the correct figure of merit for thermometry. Its full minimization provides an operational and optimal rule to postprocess measurements into a temperature reading, and it establishes a global precision limit. We apply these results to the simulated outcomes of measurements on a spin gas, finding that the local approach can lead to biased temperature estimates in cases where the global estimator converges to the true temperature. The global framework thus enables a reliable approach to data analysis in thermometry experiments.
\end{abstract}

DOI: $10.1103 /$ PhysRevLett.127.190402

Quantum thermometry aims to improve precision standards for temperature sensing in the quantum regime [1-3]. It can inform the design of nanoscale probes [4,5], the choice of measurement [6-9], and, as we shall see, the postprocessing of measured data into an optimal temperature reading. Precision thermometry is rooted in the old problem of interpreting temperature fluctuations-in practice, temperature cannot be accessed directly but rather, estimated from the statistics of observable properties [10-14]. More generally, estimation theory allows one to devise feasible strategies that can approach the fundamental precision limits of thermometry. Improving thermometric accuracy is not only relevant for quantum thermodynamics [2], but also in any practical application in which precise temperature control is necessary.

Strategies for temperature estimation may be classified as follows. Let $T$ denote a true but unknown temperature, and let $\theta \in\left[\theta_{1}, \theta_{2}\right]$ represent a hypothesis about the value of $T$. Local estimation schemes assume that $\theta_{2} / \theta_{1} \sim 1$. If no such a priori hypothesis is required, the scheme is said to be global [15]. One readily sees that, if $\theta_{2} / \theta_{1} \sim 1$ and $T \in\left[\theta_{1}, \theta_{2}\right]$, then $\theta \sim T$. Consequently, local strategies allow one to reduce statistical fluctuations once the temperature is well known [18], but they cannot address the estimation of unknown temperatures in full generality. Currently, most literature on quantum thermometry focuses on local protocols [1-9].

This is partly due to the widespread use of the CramérRao bound (CRB) $[19,20]$ as the precision benchmark. The standard CRB assumes unbiased estimators, i.e., that the temperature estimates average to $T$, and it is exactly saturable only for a special class of probability modelsthe exponential family $[18,21]$. To accommodate a wider model set, one can employ local unbiased estimators [22] which are appropriate when the unknown temperature lies initially on a very narrow interval [18]. More generally, the CRB is approached using asymptotically large data sets $[21,23]$, which in turn reduces the estimation error down to a regime in which local strategies become optimal. The applicability of the CRB thus leads to schemes that are useful only in a local sense, excluding cases where little is known about the temperature a priori, or where only few measurements can be performed. Furthermore, even if an exact saturation of the CRB were possible, the bound often depends explicitly on the unknown $T$ [3]. In general, local thermometry is thus far too restrictive.

This Letter puts forward a new method for global quantum thermometry, that is, a method applicable to estimates based on small data sets even if $\theta$ lies on a broad range. Here the problem of global temperature estimation is formulated, and fully solved, within the Bayesian framework [24-26]. We achieve this by assigning a prior probability reflecting the initial state of information about temperature, and identifying an appropriate measure of uncertainty (an averaged "cost" or "deviation"). The latter must also respect the scale invariance of the problem, and turns out to be a type of mean logarithmic error. Equipped with this measure of uncertainty, we are able to derive analytical expressions for the optimal temperature estimator and its uncertainty, neither of which assume statistical unbiasedness. Under certain conditions, local thermometry is recovered as a special case of this global formalism.

As a means of illustration, we apply the global method to a noninteracting gas of $n$ spin-1/2 particles. For this example, local thermometry is found to be unable to predict the true precision scaling when $n \lesssim 10^{7}$ (with 
5\% tolerance). Moreover, the estimator identified as optimal in the local regime can in principle yield a much larger uncertainty than its global counterpart. To demonstrate the potential usefulness of the global approach in the analysis of experimental data, we also simulate and postprocess measurement outcomes for this $n$-spin gas. The global estimator is then found to converge to the true temperature after $\mu \simeq 10^{2}$ trials. In contrast, a local analysis can lead to a biased temperature estimate even for large $\mu$. These results show that a paradigm shift toward Bayesian techniques may allow a more robust and significantly enhanced optimization of thermometric protocols, especially in cases where the data are limited [23,27,28].

Scale invariance and logarithmic error-Consider a system in equilibrium, where the true temperature $T$ is well defined but unknown. To infer it, one can perform energy measurements, which return the value $E$, given $T$, according to some conditional probability distribution. This plays the role of a likelihood function [21,24], as it links the measurement process with the unknown parameter. We denote such function by $p(E \mid \theta)$, where we recall that $\theta$ is our hypothesis for the true temperature $T$. Instead of assuming that $\theta \sim T$, as local thermometry does, we introduce a prior probability $p(\theta)$ as a proxy for our initial state of information about $T$. It is then instructive to adopt the limit of complete ignorance, opposite to (and more general than) local estimation.

Naively, one would represent complete ignorance as $p(\theta) \propto 1$. However, the conditional probability $p(E \mid \theta)$ only depends on $\theta$ through the dimensionless ratio $E /\left(k_{B} \theta\right)$, i.e.,

$$
p(E \mid \theta) d E=\frac{f\left[E /\left(k_{B} \theta\right)\right]}{\int d \hat{E} f\left[\hat{E} /\left(k_{B} \theta\right)\right]} d E
$$

for some function $f$ and where $k_{B}$ is Boltzmann's constant. This implies that we are dealing with a scale estimation problem [24,25,29]. Given that $T$ is, at this stage, completely unknown, so is the scale of the problem. Consistency thus demands our initial state of information to be invariant under transformations $E \mapsto E^{\prime}=\gamma E$ and $\theta \mapsto \theta^{\prime}=\gamma \theta[11,24]$, where $\gamma$ is a dimensionless constant. In turn, this means that the prior probability must satisfy $p(\theta) d \theta=p\left(\theta^{\prime}\right) d \theta^{\prime}$, which leads to the functional equation $p(\theta)=\gamma p(\gamma \theta)$ with solution $p(\theta) \propto 1 / \theta[24,25]$. Indeed, note that the problem could have been equivalently formulated in terms of the inverse temperature $\beta=1 / k_{B} \theta$. Such a choice should not alter our prior knowledge, and yet, taking $p(\theta) \propto 1$ gives $p(\beta) \propto 1 / \beta^{2}$, while $p(\theta) \propto 1 / \theta$ correctly leads to $p(\beta) \propto 1 / \beta[11]$.

To map a measurement outcome $E$ into a temperature, we build a point estimator $\tilde{\theta}(E)$. Its quality is assessed via some deviation function $\mathcal{D}[\tilde{\theta}(E), \theta]$ gauging the deviation of $\tilde{\theta}(E)$ from $\theta$. Since all the required information is contained in the joint probability $p(E, \theta)=p(\theta) p(E \mid \theta) \propto p(E \mid \theta) / \theta$, we write the average uncertainty of $\tilde{\theta}(E)$ as

$$
\bar{\epsilon}_{\mathcal{D}}:=\int d E d \theta p(E, \theta) \mathcal{D}[\tilde{\theta}(E), \theta] .
$$

The integration over $\theta$ accounts for all the available prior information and makes Eq. (2) temperature independent. This is a key feature that, unlike the local approach, leads to well-posed optimization problems [24].

Our next step is to establish the form of the deviation function $\mathcal{D}$. Let the dimensionless scalar $x \in(-\infty, \infty)$, and let its prior probability be $p(x) \propto 1$. As discussed, e.g., in Ref. [24], this is a translationally invariant estimation problem, for which a flat prior probability represents complete ignorance. The deviation of $\tilde{x}(E)$ from $x$ is then naturally quantified by the $k$ distance $\mathcal{D}[\tilde{x}(E), x]=$ $|\tilde{x}(E)-x|^{k}$. Now we observe that setting $x=\alpha \log \left(k_{B} \theta / \varepsilon_{0}\right)$ maps this hypothetical scenario into our thermometry problem, since $p(x) d x=p(\theta) d \theta$ implies $p(x) \propto 1 \mapsto$ $p(\theta) \propto 1 / \theta$. Here, $\varepsilon_{0}$ is an arbitrary constant with units of energy included merely for dimension neutralization [30], while $\alpha$ is a proportionality factor. Therefore,

$$
\mathcal{D}[\tilde{x}(E), x] \mapsto \mathcal{D}[\tilde{\theta}(E), \theta]=\left|\alpha \log \left[\frac{\tilde{\theta}(E)}{\theta}\right]\right|^{k},
$$

which is a bona fide scale parameter deviation function: it is symmetric, i.e., $\mathcal{D}(\tilde{\theta}, \theta)=\mathcal{D}(\theta, \tilde{\theta})$; it respects the invariance of the problem, that is, $\mathcal{D}(\gamma \tilde{\theta}, \gamma \theta)=\mathcal{D}(\tilde{\theta}, \theta)$; it reaches its absolute minimum at $\tilde{\theta}=\theta$ where it vanishes; and it grows (decreases) monotonically from (toward) that minimum when $\tilde{\theta}>\theta(\tilde{\theta}<\theta)$. While there may be other functions compatible with these conditions, Eq. (3) is certainly a suitable choice for thermometry. Below we further show that for $\alpha=1$ and $k=2$, the global framework can be reduced to local thermometry assuming one does have prior (local) information. For that reason, we will fix $\alpha=1$ and $k=2$ in the following, and after Eq. (3) is inserted in Eq. (2), we arrive at

$$
\bar{\epsilon}_{\mathrm{mle}}=\int d E d \theta p(E, \theta) \log ^{2}\left[\frac{\tilde{\theta}(E)}{\theta}\right] .
$$

We call $\bar{\epsilon}_{\text {mle }}$ the mean logarithmic error.

Optimal global strategy.-Our goal is to find the temperature estimator that is optimal with respect to Eq. (4). To do this, we must minimize $\bar{\epsilon}_{\text {mle }}$ over all possible estimators. Since $\bar{\epsilon}_{\text {mle }}$ is a functional of $\tilde{\theta}(E)$, i.e., $\bar{\epsilon}_{\text {mle }}=\epsilon[\tilde{\theta}(E)]$, this is achieved by solving the variational problem

$$
\delta \epsilon[\tilde{\theta}(E)]=\delta \int d E \mathcal{L}[\tilde{\theta}(E), E]=0,
$$

where $\mathcal{L}[\tilde{\theta}(E), E]:=\int d \theta p(E, \theta) \log ^{2}[\tilde{\theta}(E) / \theta]$ plays the role of a Lagrangian. We find that the optimal estimator $\tilde{\vartheta}(E)$ minimizing Eq. (4) is given by

$$
\frac{k_{B} \tilde{\vartheta}(E)}{\varepsilon_{0}}=\exp \left[\int d \theta p(\theta \mid E) \log \left(\frac{k_{B} \theta}{\varepsilon_{0}}\right)\right],
$$


where $p(\theta \mid E)=p(E, \theta) / p(E)$ is the posterior probability, given by Bayes theorem, and $p(E)=\int d \theta p(E, \theta)$ [31]. Inserting the optimal estimator $\tilde{\vartheta}(E)$ as $\tilde{\theta}(E)$ in Eq. (4) further gives the optimal logarithmic error $\bar{\epsilon}_{\text {opt }}$. The latter can be interpreted intuitively when split as

$$
\bar{\epsilon}_{\mathrm{opt}}=\bar{\epsilon}_{p}-\mathcal{K},
$$

where $\bar{\epsilon}_{p}:=\int d \theta p(\theta) \log ^{2}\left(\tilde{\vartheta}_{p} / \theta\right)$ is the uncertainty prior to any measurement, with optimal (prior) estimate $k_{B} \tilde{\vartheta}_{p} / \varepsilon_{0}=\exp \left[\int d \theta p(\theta) \log \left(k_{B} \theta / \varepsilon_{0}\right)\right]$, and

$$
\mathcal{K}:=\int d E p(E) \log ^{2}\left[\frac{\tilde{\vartheta}(E)}{\tilde{\vartheta}_{p}}\right]
$$

can be thought of as the maximal information provided by the measurement $E$, on average. The calculations leading to Eqs. (6) and (7) are given in the Supplemental Material [32].

Eqs. (6) and (7) constitute our main result. The former gives an optimal estimator $\tilde{\vartheta}(E)$ requiring no prior assumptions and directly applicable on a given dataset. Equation (7) indicates the corresponding uncertainty $\bar{\epsilon}_{\text {opt }}$. Since $\bar{\epsilon}_{\text {opt }}$ is a true minimum, Eq. (7) also serves as a generalized precision "bound" for temperature estimation [36]. Unlike the CRB [3], this holds for any estimator, biased or unbiased. In addition, these results, currently written for a single measurement with outcome $E$, can be trivially adapted to account for any number of repetitions, i.e., $\left(E_{1}, E_{2}, \ldots\right)$ with identically and independently distributed statistics [27]. Global thermometry is thus able to build temperature estimates drawn from arbitrary data sets, including cases with scarce data.

Recovery of local thermometry.-While, for the sake of generality, $T$ was initially assumed to be completely unknown, one may insert a more localized prior probability $p(\theta)$ from Eq. (4) onward [37,38]. In that case, the hypothesis $\theta$ will effectively lie in a narrow range, and the estimator $\tilde{\theta}(E)$ will be close to $\theta$. One then has $\log ^{2}[\tilde{\theta}(E) / \theta] \simeq[\tilde{\theta}(E) / \theta-1]^{2}$, so that Eq. (4) can be approximated as

$$
\bar{\epsilon}_{\mathrm{mle}} \simeq \int d \theta p(\theta) \frac{\Delta \tilde{\theta}^{2}}{\theta^{2}}
$$

where $\Delta \tilde{\theta}^{2}:=\int d E p(E \mid \theta)[\tilde{\theta}(E)-\theta]^{2}$. Equation (9) is the averaged noise-to-signal ratio $\Delta \tilde{\theta}^{2} / \theta^{2}$ weighted by the prior probability [39]. Since $\Delta \tilde{\theta}^{2}$ is the "frequentist" mean square error [18], it may be lower bounded as [21,24]

$$
\Delta \tilde{\theta}^{2} \geqslant \frac{1}{F(\theta)}\left[1+\frac{\partial b(\theta)}{\partial \theta}\right]^{2}+b(\theta)^{2}
$$

where we have introduced the Fisher information

$$
F(\theta)=\int \frac{d E}{p(E \mid \theta)}\left[\frac{\partial p(E \mid \theta)}{\partial \theta}\right]^{2}
$$

and the bias $b(\theta):=\int d E p(E \mid \theta)[\tilde{\theta}(E)-\theta]$. Provided that $b(\theta) \simeq 0$ as is assumed in the local approach [18], we see that Eqs. (9) and (10), and $p(\theta) / \theta^{2} \geqslant 0$ lead to the CramérRao-like bound

$$
\bar{\epsilon}_{\mathrm{mle}} \gtrsim \int d \theta \frac{p(\theta)}{\theta^{2} F(\theta)}:=\bar{\epsilon}_{\mathrm{CR}} .
$$

Equality would hold only inasmuch as the assumptions underpinning Eq. (12) are fulfilled. Namely, $b(\theta) \simeq 0$ and closeness of $\tilde{\theta}(E)$ and $\theta$, which makes $\bar{\epsilon}_{\mathrm{CR}}$ a local quantifier even when $\theta$ is integrated. Hence, we have derived a local form of thermometry as a limit of the global framework.

Note that the quantifier $\bar{\epsilon}=\int d \theta p(\theta) F(\theta)^{-1}$ has been proposed as an attempt to supersede the local paradigm [41,42]. Notwithstanding its merits-Ref. [42] reports results beyond standard local thermometry-such $\bar{\epsilon}$ is not scale invariant. This might be ignored if the prior probability is narrowly concentrated around some fixed $\theta^{\prime}$, since then $\bar{\epsilon}_{\mathrm{CR}} \simeq\left[\left(\theta^{\prime}\right)^{2} F\left(\theta^{\prime}\right)\right]^{-1}$ and $\bar{\epsilon} \simeq F\left(\theta^{\prime}\right)^{-1}$. That is, one is left in both cases with the Fisher information. But this limiting assumption is unnecessary in our truly global and intrinsically scale invariant approach.

Example: Noninteracting spin gas.-We now turn to illustrate how to find the best thermometric precision one could possibly get. Let us consider a gas of $n$ noninteracting spin- $1 / 2$ particles with energy gap $\hbar \omega$ in thermodynamic equilibrium. This could correspond, e.g., to a dilute cloud of impurities fully equilibrated with a cotrapped ultracold majority gas, whose temperature needs to be measured precisely [43-46]. The probability of measuring the total energy $E$ to be $r \hbar \omega$, with $r=0,1, \ldots, n$, is $[12,47]$

$$
p(r \mid \theta)=\left(\begin{array}{c}
n \\
r
\end{array}\right) \frac{\exp \left[-r \hbar \omega /\left(k_{B} \theta\right)\right]}{Z\left[\hbar \omega /\left(k_{B} \theta\right)\right]}
$$

where $Z\left[\hbar \omega /\left(k_{B} \theta\right)\right]=\left\{\exp \left[-\hbar \omega /\left(k_{B} \theta\right)\right]+1\right\}^{n}$ is the partition function. Additionally, suppose that $p(\theta) \propto 1 / \theta$ is defined, for instance, on the finite support $k_{B} \theta /(\hbar \omega) \in$ $[0.1,10]$, so that normalization gives $p(\theta)=1 /[2 \theta \log (10)]$.

The optimum $\bar{\epsilon}_{\text {opt }}$ can be readily evaluated after inserting the prior probability $p(\theta)$ and the likelihood [Eq. (13)] into Eq. (7) [48]. The result, for $n$ ranging from 10 to $10^{5}$, is shown in Fig. 1(a). The local limit of this error [cf. Fig. 1(a)] is shown to take the form $\bar{\epsilon}_{\mathrm{CR}} \simeq 51.7 / n$ in the Supplemental Material. Comparing both, we observe convergence as $n \rightarrow \infty$, confirming the emergence of local thermometry within the global framework. However, for finite $n$ we see that $\bar{\epsilon}_{\mathrm{CR}}>\bar{\epsilon}_{\mathrm{opt}}$. That is, while the global estimator extracts all available information for any $n$, a local approach leads to information loss when $n$ is small. 

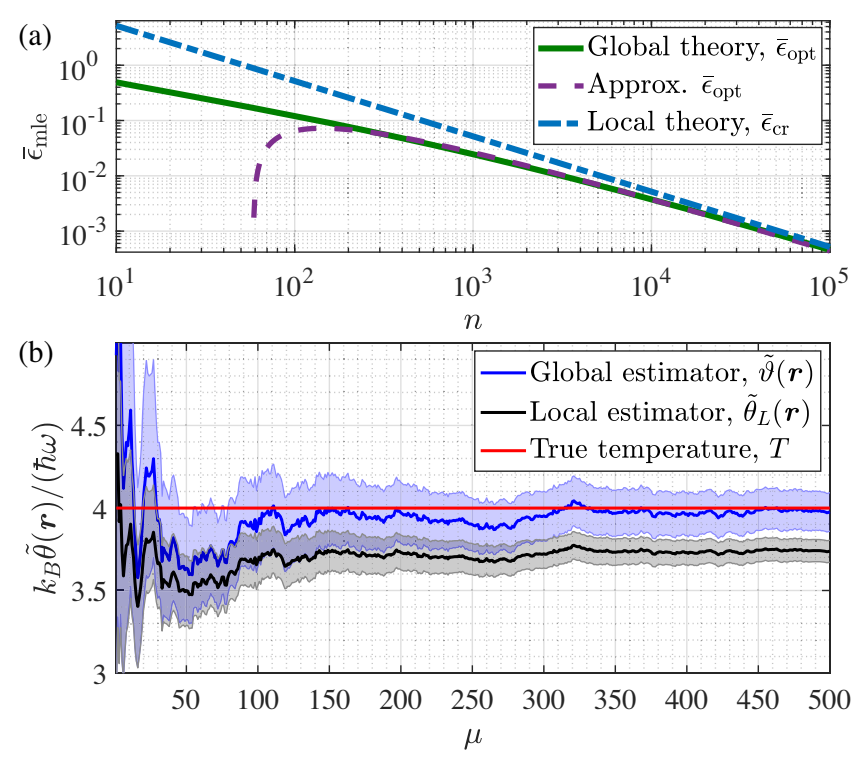

FIG. 1. (a) Log-log plot of the global optimum $\bar{\epsilon}_{\text {opt }}$ in Eq. (7) (solid green) and local Cramér-Rao-like bound in Eq. (12) (dotdashed blue) for a gas of $n$ noninteracting spin-1/2 particles in thermal equilibrium. As can be seen, the global optimum is lower than the local bound unless $n \rightarrow \infty$, meaning that the local bound misses information when $n$ is "too small." Eq. (14) gives an asymptotic expansion matching the global optimum when $n \gtrsim 10^{2}$ (dashed purple). (b) Data analysis in global thermometry. We simulated the outcomes $\boldsymbol{r}=\left(r_{1}, \ldots, r_{\mu}\right)$ of $\mu$ energy measurements on the $n$-spin gas, with $n=150$ and true temperature (solid red) $k_{B} T /(\hbar \omega)=4$. We then postprocessed $\boldsymbol{r}$ using the global estimator in Eq. (6) (dotted blue) and a local estimator initialized at $k_{B} \theta_{0} /(\hbar \omega)=3[18,21]$ (dot-dashed black). The global estimate converges to the true temperature after $\mu \simeq 10^{2}$ shots. In contrast, the local method leads to a biased estimate even for $\mu \simeq 500$. The global error is calculated via Eq. (4) but with average over the posterior $p(\theta \mid \boldsymbol{r})$ (see Supplemental Material). The standard CRB gives the local error $[18,21]$.

To gain analytical insight into the scaling of $\bar{\epsilon}_{\text {opt }}$ with $n$, first we note that, in this specific example, $\bar{\epsilon}_{\mathrm{CR}}-\bar{\epsilon}_{\mathrm{opt}} \simeq b n^{q}$. A numerical fit [49] renders the asymptotic expansion

$$
\bar{\epsilon}_{\mathrm{opt}} \simeq \frac{51.7}{n}-\frac{143}{n^{5 / 4}},
$$

which, as shown in Fig. 1(a), matches the true optimum $\bar{\epsilon}_{\text {opt }}$ when $n \gtrsim 10^{2}$. This in turn allows one to assess how large $n$ needs to be for the (local) $\sim 1 / n$ scaling to hold approximately. Consider the equation $\left|\bar{\epsilon}_{\mathrm{CR}}[n(\tau)]-\bar{\epsilon}_{\text {opt }}[n(\tau)]\right|=$ $\tau \bar{\epsilon}_{\text {opt }}[n(\tau)]$ [38], where $n(\tau)$ is the minimum number of spins which drives the relative error between the optimal and asymptotic curves below a tolerance $\tau$. Using Eq. (14) gives the condition $n(\tau) \simeq 58.5(1+1 / \tau)^{4}$. As expected, $n(\tau) \rightarrow \infty$ when $\tau \rightarrow 0$. But, even when tolerating a $5 \%$ deviation, spin numbers of $n \sim 10^{7}$ are required for local thermometry to give a correct scaling. Admittedly, the value of $n(\tau)$ is protocol dependent $[23,38]$, but even a simple example suffices to illustrate the perils of the local frameworkinformation loss [Fig. 1(a)] and failure to produce a valid low- $n$ scaling [Eq. (14)].

Data analysis in global thermometry.-The global framework does not only enable a more comprehensive picture of fundamental limits, but, perhaps more importantly, is also a reliable tool for experimental data analysis. Consider the following protocol: a gas of $n$ spin- $1 / 2$ particles is prepared; its energy $r \hbar \omega$ is measured; both steps are repeated $\mu$ times, generating the outcomes $\left(r_{1}, r_{2}, \ldots, r_{\mu}\right):=r$. The global estimate $\tilde{\vartheta}(\boldsymbol{r})$ in Eq. (6) is calculated using the posterior $p(\theta \mid \boldsymbol{r}) \propto p(\boldsymbol{r} \mid \theta) / \theta$, with $k_{B} \theta /(\hbar \omega) \in[0.1,10], \quad p(\boldsymbol{r} \mid \theta)=$ $\prod_{i=1}^{\mu} p\left(r_{i} \mid \theta\right)$ and $p\left(r_{i} \mid \theta\right)$ given by Eq. (13). To assess its uncertainty, the average over $p(\boldsymbol{r}, \theta)$ in Eq. (4) is instead taken over $p(\theta \mid \boldsymbol{r})$, since, in experiments, the outcomes $\boldsymbol{r}$ are known [24,26]. The resulting error $\bar{\epsilon}_{\text {mle }}(\boldsymbol{r})$ is obviously outcome dependent, while being temperature independent [50]. Recalling that the logarithmic error is a noise-to-signal ratio, we introduce the Bayesian "error bar" $\Delta \tilde{\theta}(\boldsymbol{r}):=$ $\tilde{\theta}(\boldsymbol{r}) \sqrt{\bar{\epsilon}_{\text {mle }}(\boldsymbol{r})}$. This analysis may be compared with the local CRB-based estimator $\tilde{\theta}(\boldsymbol{r})_{L}=\theta_{0}+\left[\mu F\left(\theta_{0}\right)\right]^{-1} \partial \log \left[p\left(\boldsymbol{r} \mid \theta_{0}\right)\right] /$ $\partial \theta$, with error $\Delta \tilde{\theta}_{L}=1 / \sqrt{\mu F\left(\theta_{0}\right)}[18,21]$. Here, $\theta_{0}$ is an initial "hint" at the true temperature $T$, a prerequisite in local thermometry.

We simulated the outcomes $\boldsymbol{r}$ arising from the aforementioned protocol, for a gas with $n=150$ spins and true temperature $k_{B} T /(\hbar \omega)=4$. The local estimate $\tilde{\theta}_{L} \pm \Delta \tilde{\theta}_{L}$, initialized with the "hint" $k_{B} \theta_{0} /(\hbar \omega)=3$, is shown in Fig. 1(b) (dot-dashed black) to be biased even when $\mu \gg 1$. This contrasts with the convergence of the global estimate $\tilde{\vartheta} \pm \Delta \tilde{\vartheta}$ (dotted blue) to the true temperature (solid red). One could argue for a "two-step" method where a part of the data is used to provide a better $\theta_{0}$ prior to applying local thermometry. Yet, one cannot anticipate how many trials a good seed $\theta_{0}$ requires, nor when such $\theta_{0}$ is sufficiently close to the true temperature. The global framework is instead general, reliable, and works at once. Further evidence is provided in the Supplemental Material, where a comparison between global thermometry and a histogram-fitting procedure demonstrates the potential gain of global techniques for experiments with limited data.

Conclusions. - We have demonstrated that local precision benchmarks are insufficient whenever few data or no substantial prior knowledge are available. On the contrary, a global approach is applicable to any temperatureestimation protocol regardless of the measurement record length, and can naturally account for any degree of prior information. For instance, it would be interesting to exploit Eq. (6) to postprocess data measured in the nanokelvin regime, which is experimentally accessible with ultracold Bose and Fermi gases [43,44,51-55] and relevant for quantum simulation [56]. In addition, since Eq. (4) is deduced at the level of probability distributions [i.e., with no explicit consideration of the Born rule $p(E \mid \theta)=\operatorname{Tr}[\Pi(E) \rho(\theta)]$ 
[57] ], Eq. (6) can be applied also to classical systems. Finally, note that the key assumption behind Eq. (4) is that the parameter is a scale, which makes it applicable beyond temperature estimation (e.g., to estimate biochemical rates in single-molecule experiments [58-61]).

From a fundamental perspective, combining Eq. (6) the rule to calculate optimal estimates-with the theoretical optimum [Eq. (7)] provides a powerful tool to address open problems in thermometry. These include pushing precision limits further by optimizing over the energy spectrum of the probe system $[4,42]$, or over measured quantities beyond energy (see Supplemental Material). Moreover, the global formalism may be extended to accommodate for the nonequilibrium states $[8,62,63]$ resulting from limited access to interacting thermalized probes, and gives the theoretical support needed to derive more fundamental energytemperature uncertainty relations [64].

We thank A. Bayat, W.-K. Mok, K. Bharti, M. PerarnauLlobet, A. Luis, and S. Subramanian for helpful discussions. J. R. and J. A. acknowledge support from EPSRC (Grant No. EP/T002875/1), and J. A. acknowledges support from EPSRC (Grant No. EP/R045577/1) and the Royal Society.

*J.Rubio-Jimenez@exeter.ac.uk

[1] T. M. Stace, Quantum limits of thermometry, Phys. Rev. A 82, 011611(R) (2010).

[2] A. De Pasquale and T. M. Stace, Quantum thermometry, in Thermodynamics in the Quantum Regime: Fundamental Aspects and New Directions, edited by F. Binder, L. A. Correa, C. Gogolin, J. Anders, and G. Adesso (Springer International Publishing, Cham, 2018), pp. 503-527.

[3] M. Mehboudi, A. Sanpera, and L. A. Correa, Thermometry in the quantum regime: Recent theoretical progress, J. Phys. A 52, 303001 (2019).

[4] L. A. Correa, M. Mehboudi, G. Adesso, and A. Sanpera, Individual Quantum Probes for Optimal Thermometry, Phys. Rev. Lett. 114, 220405 (2015).

[5] M. Płodzień, R. Demkowicz-Dobrzański, and T. Sowiński, Few-fermion thermometry, Phys. Rev. A 97, 063619 (2018).

[6] M. Mehboudi, M. Moreno-Cardoner, G. De Chiara, and A. Sanpera, Thermometry precision in strongly correlated ultracold lattice gases, New J. Phys. 17, 055020 (2015).

[7] L. A. Correa, M. Perarnau-Llobet, K. V. Hovhannisyan, S. Hernández-Santana, M. Mehboudi, and A. Sanpera, Enhancement of low-temperature thermometry by strong coupling, Phys. Rev. A 96, 062103 (2017).

[8] P. P. Potts, J. B. Brask, and N. Brunner, Fundamental limits on low-temperature quantum thermometry with finite resolution, Quantum 3, 161 (2019).

[9] M. T. Mitchison, T. Fogarty, G. Guarnieri, S. Campbell, T. Busch, and J. Goold, In Situ Thermometry of a Cold Fermi Gas Via Dephasing Impurities, Phys. Rev. Lett. 125, 080402 (2020).
[10] G. D. J. Phillies, The polythermal ensemble: A rigorous interpretation of temperature fluctuations in statistical mechanics, Am. J. Phys. 52, 629 (1984).

[11] H. B. Prosper, Temperature fluctuations in a heat bath, Am. J. Phys. 61, 54 (1993).

[12] T. Jahnke, S. Lanéry, and G. Mahler, Operational approach to fluctuations of thermodynamic variables in finite quantum systems, Phys. Rev. E 83, 011109 (2011).

[13] M. Falcioni, D. Villamaina, A. Vulpiani, A. Puglisi, and A. Sarracino, Estimate of temperature and its uncertainty in small systems, Am. J. Phys. 79, 777 (2011).

[14] J. Leo van Hemmen and A. Longtin, Temperature fluctuations for a system in contact with a heat bath, J. Stat. Phys. 153, 1132 (2013).

[15] 'Local' and 'global' are here defined with respect to the space of hypotheses for the true temperature, as is customary in quantum estimation theory [16]. However, the same terminology has been given different meaning when probes are multipartite: states and measurements associated with a part of the whole are said to be local, and global otherwise. These notions of 'local' and 'global' can also be found in quantum thermometry [2,17]. However, the version of estimation theory used is still local in the standard sense, i.e., with respect to the parameter space. Therefore, the spatial partitioning of the probe is not relevant to the present discussion.

[16] M. G. A. paris, Quantum estimation for quantum technology, Int. J. Quantum. Inform. 07, 125 (2009).

[17] S. Campbell, M. Mehboudi, G. D. Chiara, and M. Paternostro, Global and local thermometry schemes in coupled quantum systems, New J. Phys. 19, 103003 (2017).

[18] R. Demkowicz-Dobrzański, M. Jarzyna, and J. Kołodyński, Quantum limits in optical interferometry, Prog. Opt. 60, 345 (2015).

[19] H. Cramér, Mathematical Methods of Statistics (Princeton University Press, Princeton, NJ, 1999), Vol. 43.

[20] C. R. Rao, Information and the accuracy attainable in the estimation of statistical parameters, in Breakthroughs in statistics (Springer, New York, 1992), pp. 235-247.

[21] S. Kay, Fundamentals of Statistical Signal Processing: Estimation Theory (Prentice Hall, Englewood Cliffs, NJ, 1993).

[22] D. A. S. Fraser, On local unbiased estimation, J. R. Stat. Soc. Ser. B 26, 46 (1964).

[23] J. Rubio and J. A. Dunningham, Quantum metrology in the presence of limited data, New J. Phys. 21, 043037 (2019).

[24] E. T. Jaynes, Probability Theory: The Logic of Science (Cambridge University Press, Cambridge, England, 2003).

[25] U. von Toussaint, Bayesian inference in physics, Rev. Mod. Phys. 83, 943 (2011).

[26] J. Rubio Jiménez, Non-asymptotic quantum metrology: Extracting maximum information from limited data, Ph.D. thesis, University of Sussex, 2020.

[27] J. Rubio and J. Dunningham, Bayesian multiparameter quantum metrology with limited data, Phys. Rev. A 101, 032114 (2020).

[28] S. Morelli, A. Usui, E. Agudelo, and N. Friis, Bayesian parameter estimation using Gaussian states and measurements, Quantum Sci. Technol. 6, 025018 (2021).

[29] R. E. Kass and L. Wasserman, The selection of prior distributions by formal rules, J. Am. Stat. Assoc. 91, 1343 (1996). 
[30] C. F. Matta, L. Massa, A. V. Gubskaya, and E. Knoll, Can one take the logarithm or the sine of a dimensioned quantity or a unit? Dimensional analysis involving transcendental functions, J. Chem. Educ. 88, 67 (2011).

[31] Note that Eq. (6) is independent of the specific value of $k_{B} / \varepsilon_{0}$.

[32] See Supplemental Material at http://link.aps.org/ supplemental/10.1103/PhysRevLett.127.190402 for further details on the derivations, simulations and examples in the main text, which includes Refs. [33-35].

[33] K. F. Riley, M. P. Hobson, and S. J. Bence, Mathematical Methods for Physics and Engineering (Cambridge University Press, Cambridge, England, 2004).

[34] R. K. Pathria, Statistical Mechanics, 3rd ed. (Elsevier, Amsterdam, 2011).

[35] M. Hohmann, F. Kindermann, T. Lausch, D. Mayer, F. Schmidt, and A. Widera, Single-atom thermometer for ultracold gases, Phys. Rev. A 93, 043607 (2016).

[36] An analogous result exists for multi-phase estimation [27].

[37] R. Demkowicz-Dobrzański, Optimal phase estimation with arbitrary a priori knowledge, Phys. Rev. A 83, 061802(R) (2011).

[38] J. Rubio, P. A. Knott, and J. A. Dunningham, Non-asymptotic analysis of quantum metrology protocols beyond the CramérRao bound, J. Phys. Commun. 2, 015027 (2018).

[39] Interestingly, $\log ^{2}[\tilde{\theta}(E) / \theta]$ and $[\tilde{\theta}(E)-\theta]^{2} / \theta^{2}$, which give rise to Eqs. (4) and (9), respectively, have been previously considered as independent choices with different properties [40]. Instead, here we derive Eq. (4) by imposing physically motivated constraints, while Eq. (9) appears as a local limiting case. Hence, we may regard the former as more fundamental.

[40] J. G. Norstrom, The use of precautionary loss functions in risk analysis, IEEE Trans. Reliab. 45, 400 (1996).

[41] M. E. Pearce, E. T. Campbell, and P. Kok, Optimal quantum metrology of distant black bodies, Quantum 1, 21 (2017).

[42] W.-K. Mok, K. Bharti, L.-C. Kwek, and A. Bayat, Optimal probes for global quantum thermometry, Commun. Phys. 4, 1 (2021).

[43] Q. Bouton, J. Nettersheim, D. Adam, F. Schmidt, D. Mayer, T. Lausch, E. Tiemann, and A. Widera, Single-Atom Quantum Probes for Ultracold Gases Boosted by Nonequilibrium Spin Dynamics, Phys. Rev. X 10, 011018 (2020).

[44] R. Olf, F. Fang, G. E. Marti, A. MacRae, and D. M. Stamper-Kurn, Thermometry and cooling of a Bose gas to 0.02 times the condensation temperature, Nat. Phys. 11, 720 (2015).

[45] M. Mehboudi, A. Lampo, C. Charalambous, L. A. Correa, M. A. García-March, and M. Lewenstein, Using Polarons for sub-nK Quantum Nondemolition Thermometry in a BoseEinstein Condensate, Phys. Rev. Lett. 122, 030403 (2019).

[46] M. T. Mitchison, T. Fogarty, G. Guarnieri, S. Campbell, T. Busch, and J. Goold, In Situ Thermometry of a Cold Fermi Gas via Dephasing Impurities, Phys. Rev. Lett. 125, 080402 (2020).

[47] G. A. P. Wyllie, Elementary Statistical Mechanics (Hutchinson, London, 1970).
[48] Numerical algorithms on GitHub https://github.com/jesusrubiojimenez/QuThermometry-global (2020).

[49] Specifically, a Bayesian fit using $\log \left[\bar{\epsilon}_{\mathrm{cr}}(n)-\bar{\epsilon}_{\mathrm{opt}}(n)\right]=$ $q \log (n)+\log (b)+e(n)$, with Gaussian errors $e(n)$.

[50] In the Supplemental Material we prove that Eq. (6) is optimal also with respect to $\bar{\epsilon}_{\text {mle }}(\boldsymbol{r})$.

[51] J. Javanainen and J. Ruostekoski, Off-resonance light scattering from low-temperature Bose and Fermi gases, Phys. Rev. A 52, 3033 (1995).

[52] A. Leanhardt, T. Pasquini, M. Saba, A. Schirotzek, Y. Shin, D. Kielpinski, D. Pritchard, and W. Ketterle, Cooling BoseEinstein condensates below 500 picokelvin, Science 301, 1513 (2003).

[53] J. Ruostekoski, C. J. Foot, and A. B. Deb, Light Scattering for Thermometry of Fermionic Atoms in an Optical Lattice, Phys. Rev. Lett. 103, 170404 (2009).

[54] R. Onofrio, Cooling and thermometry of atomic Fermi gases, Phys. Usp. 59, 1129 (2016).

[55] C. Carcy, G. Hercé, A. Tenart, T. Roscilde, and D. Clément, Certifying the Adiabatic Preparation of Ultracold Lattice Bosons in the Vicinity of the Mott Transition, Phys. Rev. Lett. 126, 045301 (2021).

[56] I. Bloch, J. Dalibard, and S. Nascimbene, Quantum simulations with ultracold quantum gases, Nat. Phys. 8, 267 (2012).

[57] Here $\Pi$ denotes a probability-operator measurement (POM), while $\rho$ is a state operator.

[58] M. D. Baaske, M. R. Foreman, and F. Vollmer, Singlemolecule nucleic acid interactions monitored on a labelfree microcavity biosensor platform, Nat. Nanotechnol. 9, 933 (2014).

[59] S. Subramanian, S. Frustaci, and F. Vollmer, Microsecond single-molecule enzymology using plasmonically enhanced optical resonators, in Frontiers in Biological Detection: From Nanosensors to Systems XII, edited by A. Danielli, B. L. Miller, and S. M. Weiss, International Society for Optics and Photonics Vol. 11258 (SPIE, 2020), pp. 23-30, https://doi.org/10.1117/12.2559381.

[60] S. Subramanian, H. Jones, S. Frustaci, S. Winter, M. van der Kamp, V. Arcus, C. Pudney, and F. Vollmer, Sensing enzyme activation heat capacity at the single-molecule level using gold-nanorod-based optical whispering gallery modes, ACS Appl. Nano Mater. 4, 4576 (2021).

[61] N. Eerqing, S. Subramanian, J. Rubio, T. Lutz, H.-Y. Wu, J. Anders, C. Soeller, and F. Vollmer, Comparing transient oligonucleotide hybridization kinetics using DNA-PAINT and optoplasmonic single-molecule sensing on gold nanorods, ACS Photonics 8, 2882 (2021).

[62] A. De Pasquale, D. Rossini, R. Fazio, and V. Giovannetti, Local quantum thermal susceptibility, Nat. Commun. 7, 12782 (2016).

[63] K. V. Hovhannisyan and L. A. Correa, Measuring the temperature of cold many-body quantum systems, Phys. Rev. B 98, 045101 (2018).

[64] H. J. D. Miller and J. Anders, Energy-temperature uncertainty relation in quantum thermodynamics, Nat. Commun. 9, 2203 (2018). 